\title{
Áreas Complementarias para la Conservación de la Flora de Preocupación Especial en Regiones Semiáridas de Honduras
}

\author{
Cindy Melissa Midence Raudales ${ }^{1}$
}

Resumen. Las formaciones vegetales secas forman parte de los ecosistemas más amenazados en el mundo. En Honduras, los bosques secos representan el $9.4 \%$ de su cobertura original producto del cambio del uso de suelo por actividades antrópicas. Como un intento de averiguar el área potencial de distribución de especies de flora de preocupación especial e identificar las áreas complementarias para su conservación basada en sitios de mayor riqueza, adentro de la ecorregión de Bosque Seco de Honduras, se aplicó un programa de modelaje bioclimático basado únicamente en presencias, generando mediante MaxEnt modelos individuales de hábitat para 27 especies. Como resultado del análisis de complementariedad se proponen tres áreas prioritarias para la conservación, siguiendo el criterio de conservar el menor número de áreas que alberguen el mayor número de especies. Se concluye que el modelo mostró su eficacia para establecer áreas de conservación, en tres áreas: Oropolí, Tegucigalpa y Morocelí situadas en la región centro y sur de Honduras.

Palabras clave: Conservación, bosque seco, distribución potencial, MaxEnt.

\section{Complementary Areas for the Conservation of Flora of Special Concern in Semi-Arid Regions of Honduras}

\begin{abstract}
Semi-arid regions are among the most threatened ecosystem in the world. In Honduras, these areas account for $9.4 \%$ of its original cover due to changes in land use by human activities. In an attempt to document the potential flora of concern in these regions of concern and identify additional areas for conservation based on richer sites, in the dry forest ecoregion in Honduras. A bioclimatic modeling program based only on presence, generating by MaxEnt individual models of habitat for 27 species was applied. As a result of the analysis of complementarity, three priority areas for conservation are proposed, following the criteria of preserving the fewest number of areas having the greatest number of species. It is confirmed that the model demonstrated its effectiveness to identify conservation areas, in the areas of: Oropolí, Tegucigalpa and Morocelí. These areas are located in the central and southern region of Honduras.
\end{abstract}

Keywords: Conservation, dry forest, potential distribution, MaxEnt.

\section{Introducción}

Cerca del $42 \%$ de los hábitats tropicales y subtropicales son bosques secos (BS) (Murphy y Lugo 1995), los cuales se distinguen por su riqueza y el alto nivel de endemismo que albergan (Trejo 2000). Los BS neotropicales se distribuyen en forma fragmentada desde el occidente de México hasta el norte de Argentina (Prado y Gibbs 1993; Miles et al. 2006). Lamentablemente, a lo largo del neotrópico, estos bosques están desapareciendo debido en parte a la ocupación de asentamientos humanos (Maass et al. 1995), la extracción de árboles de alto valor comercial, el remplazo de tierras para pastoreo, la agricultura extensiva (Miles et al. 2006) y la recurrencia de fuegos.

Honduras es parte de la región mesoamericana, aquí convergen ecosistemas con mezclas de estructuras vegetales provenientes de la región norte y sur de América desde hace unos 3 millones de años (Marshal et al. 1982). Las características climáticas y geomorfológicas de Honduras (Pineda 1997) impactan la distribución de la ecorregión seca desde la zona litoral pacífica, a través de valles intermontanos, hasta la zona litoral atlántica. La ecorregión de BS en Honduras incluye los BS con árboles gigantes que alcanzan $40 \mathrm{~m}$ por la humedad casi permanente al estar expuestos a los vientos alisios de la zona litoral y los bosques muy secos (bms) ubicados a sotavento de las cadenas montañosas, lo que produce una vegetación compuesta por arbustos y árboles entre 3 y $12 \mathrm{~m}$ casi $100 \%$ deciduos en la estación seca. La parte tropical seca incluye tres de las cuatro formaciones

\footnotetext{
${ }^{1}$ Bióloga con M.Sc. en Biodiversidad en Áreas Tropicales y su Conservación, Calle San Juan número 9, Tebar, Cuenca, España. Correo electrónico: biocmidence@yahoo.es
} 
secas para Centroamérica (Holdridge 1962): La precipitación en el bosque seco Tropical es 1000-2000 $\mathrm{mm}$, en el bosque seco Subtropical es $500-1000 \mathrm{~mm}$ y en el bosque muy seco Tropical 250-500 mm.

Honduras tiene un área de $112.492 \mathrm{~km}^{2}$ y posee 7,524 especies de plantas vasculares, de las cuales 244 son endémicas o de distribución limitada y 35 están categorizadas como especies raras 0 amenazadas (Mejía y House 2008). La Dirección de Biodiversidad de la Secretaria de Recursos Naturales y Ambiente de Honduras consideró que "Especies de Preocupación Especial en Honduras" (EPE) son las que se encuentran en las siguientes categorías: (1) Endémicas: especies de Flora y Fauna de distribución restringida y que solo se encuentran en el territorio hondureño; (2) Mesoendémicas: especies de Flora y Fauna de distribución restringida en una región compartida entre dos o tres países de Centroamérica. El listado no contempla exhaustivamente todas las especies de este grupo; (3) Especies listadas en el UICN y (4) Especies listadas en el apéndices CITES (I, II y III según el caso) (Mejía y House 2008). Basado en la lista de la IUCN (2007), 110 especies ocupan algún criterio de amenaza dentro de las categorías para especies amenazadas de extinción (Vulnerable $=30$, En Peligro $=38$, En Peligro Crítico $=42$ ). Unas 60 de estas especies pertenecen a ecosistemas deciduos en Honduras, el cual se estimaba, de acuerdo con el mapa ecológico de Honduras (Holdridge 1962), en $1,957,248$ ha, es decir, el $17.3 \%$ del país; cifra que concuerda con el mapa ecoregional elaborado por The Natural Conservancy (TNC) - World Wide Fund
Fornature (WWF) (2002). No obstante, esta ecorregión se redujo en 50 años a $185,108,765$ ha, lo que representa el $9.4 \%$ de la superficie del país (House y Midence 2007b, Midence 2010).

Considerando lo anterior, se plantearon los guientes objetivos: (1) modelar la distribución potencial de especies endémicas y amenazadas de acuerdo con la lista de EPE de Honduras, (2) estimar el porcentaje del hábitat natural perdido o transformado para cada especie, (3) obtener el mapa de riqueza de especies endémicas y amenazadas para el BS de Honduras y (4) delimitar áreas complementarias para la conservación.

\section{Métodos}

Datos Geográficos. Se elaboró una base de datos de 27 especies de la flora endémica y amenazada de Honduras basada en el listado de EPE en Honduras (Mejía y House 2008) y la Memoria del taller "Preparación de una estrategia para la conservación de especies crítica y globalmente amenazadas en Mesoamérica Norte" (García et al. 2006). Las localidades de distribución fueron obtenidas del registro de datos de distribución usando como referencia la base de datos de flora endémica y amenazada de Honduras (UNAH 2001) y la base de datos florística TROPICOS (www.tropicos.org). En total se obtuvieron 242 registros de 128 localidades (Cuadro 1). De las 27 especies seleccionadas, cuatro son cactáceas (Nopalea lutea, Opuntia guatemalensis, Pereskia lychnidiflora, Stenocereus yunckerii) y una orquídea (Myrmecophila wendlandii) y son evaluadas en el Apéndice II de CITES (McGough et al. 2004).

Cuadro 1. Datos de distribución y categoría de amenaza de 27 especies de bosque seco para Honduras seleccionadas para este estudio.

\begin{tabular}{|c|c|c|c|c|c|c|c|}
\hline \multirow[t]{2}{*}{ Especie } & \multirow[t]{2}{*}{ Familia } & \multirow[b]{2}{*}{$\begin{array}{l}\text { Regis- } \\
\text { tros }\end{array}$} & \multirow[b]{2}{*}{$\begin{array}{c}\text { Localidades } \\
\text { únicas } \\
\text { (municipios) }\end{array}$} & \multicolumn{4}{|c|}{ Categorías } \\
\hline & & & & $\begin{array}{l}\text { Endé- } \\
\text { micas }\end{array}$ & $\begin{array}{l}\text { Mesoen- } \\
\text { démicas }\end{array}$ & UICN & $\begin{array}{l}\text { Apéndices } \\
\text { CITES }\end{array}$ \\
\hline $\begin{array}{l}\text { Agonandra macrocarpa L.O. } \\
\text { Williams }\end{array}$ & Acanthaceae & 7 & 5 & & $\mathrm{X}$ & VU & \\
\hline Anisacanthus tetracaulis Leonard & Acanthaceae & 8 & 5 & & $\mathrm{X}$ & VU & \\
\hline Bakeridesia molinae D.M.Bates & Malvaceae & 5 & 4 & $X$ & & CR & \\
\hline Calliandra molinae Standl. & Leguminosae & 15 & 10 & & $\mathrm{X}$ & EN & \\
\hline Casearia williamsiana Sleumer & Flacourtiaceae & 9 & 5 & & $\mathrm{X}$ & $\mathrm{CR}$ & \\
\hline
\end{tabular}


Cuadro 1. Continuación

\begin{tabular}{|c|c|c|c|c|c|c|c|}
\hline \multirow[t]{2}{*}{ Especie } & \multirow[t]{2}{*}{ Familia } & \multirow[b]{2}{*}{$\begin{array}{l}\text { Regis- } \\
\text { tros }\end{array}$} & \multirow[b]{2}{*}{$\begin{array}{c}\text { Localidades } \\
\text { únicas } \\
\text { (municipios) }\end{array}$} & \multicolumn{4}{|c|}{ Categorías } \\
\hline & & & & $\begin{array}{l}\text { Endé- } \\
\text { micas }\end{array}$ & $\begin{array}{l}\text { Mesoen- } \\
\text { démicas }\end{array}$ & UICN & $\begin{array}{l}\text { Apéndices } \\
\text { CITES }\end{array}$ \\
\hline $\begin{array}{l}\text { Croton comes Standl. \& L.O. } \\
\text { Williams }\end{array}$ & Euphorbiaceae & 6 & 2 & $\mathrm{x}$ & & $\mathrm{CR}$ & \\
\hline $\begin{array}{l}\text { Guettarda sageretioides Ant. } \\
\text { Molina }\end{array}$ & Rubiaceae & 10 & 4 & $\mathrm{x}$ & & VU & \\
\hline Hechtia malvernii A.J.Gilmartin & Bromeliaceae & 6 & 4 & & $\mathrm{X}$ & EN & \\
\hline $\begin{array}{l}\text { lpomoea riparum Standl. \& L.O. } \\
\text { Williams }\end{array}$ & Convolvulaceae & 7 & 5 & $\mathrm{x}$ & & EN & \\
\hline Justicia calliantha Leonard & Acanthaceae & 13 & 3 & $\mathrm{X}$ & & VU & \\
\hline Leucaena lempirana C.E.Hughes & Leguminosae & 8 & 5 & $\mathrm{X}$ & & VU & \\
\hline Lithachne humilis Soderstr. & Poaceae & 9 & 6 & $\mathrm{X}$ & & VU & \\
\hline $\begin{array}{l}\text { Lonchocarpus minimiflorus Donn. } \\
\text { Sm }\end{array}$ & Leguminosae & 13 & 10 & & $\mathrm{x}$ & EN & \\
\hline $\begin{array}{l}\text { Lonchocarpus phlebophyllus Standl. } \\
\text { \& Steyerm }\end{array}$ & Leguminosae & 5 & 3 & & $\mathrm{x}$ & EN & \\
\hline $\begin{array}{l}\text { Lonchocarpus retiferus Standl. \& } \\
\text { L.O. Williams }\end{array}$ & Leguminosae & 3 & 3 & & $\mathrm{x}$ & EN & \\
\hline $\begin{array}{l}\text { Lonchocarpus sanctuarii Standl. \& } \\
\text { L.O. Williams. }\end{array}$ & Leguminosae & 10 & 2 & $\mathrm{x}$ & & $\mathrm{CR}$ & \\
\hline $\begin{array}{l}\text { Lonchocarpus trifolius Standl. \& } \\
\text { L.O. Williams }\end{array}$ & Leguminosae & 12 & 3 & $\mathrm{x}$ & & $\mathrm{CR}$ & \\
\hline $\begin{array}{l}\text { Lonchocarpus vallicola Stand. \& } \\
\text { F.J.Herm.) M. Sousa }\end{array}$ & Leguminosae & 18 & 5 & $\mathrm{x}$ & & $\mathrm{CR}$ & \\
\hline Machaerium nicaraguense Rudd. & Leguminosae & 10 & 3 & & $x$ & EN & \\
\hline $\begin{array}{l}\text { Myrmecophila wendlandii (Rchb.f.) } \\
\text { G.C Kenn }\end{array}$ & Orquideaceae & 8 & 5 & & $\mathrm{x}$ & & II \\
\hline Nopalea lutea Rose & Cactaceae & 15 & 12 & & $\mathrm{X}$ & & II \\
\hline $\begin{array}{l}\text { Opuntia guatemalensis Britton \& } \\
\text { Rose }\end{array}$ & Cactaceae & 17 & 8 & & $\mathrm{x}$ & & II \\
\hline Pereskia lychnidiflora DC. & Cactaceae & 10 & 8 & & $\mathrm{x}$ & & II \\
\hline $\begin{array}{l}\text { Pterocaulon pompilianum Standl. \& } \\
\text { L.Wms. }\end{array}$ & Compositae & 9 & 3 & $\mathrm{x}$ & & VU & \\
\hline $\begin{array}{l}\text { Stenocereus yunckerii (Standl.) M. } \\
\text { Bravo \& Sánchez-Mej. }\end{array}$ & Cactaceae & 3 & 2 & & $\mathrm{x}$ & & ॥ \\
\hline $\begin{array}{l}\text { Tradescantia velutina Kunth. \& C.D. } \\
\text { Bouché }\end{array}$ & Commelinaceae & 3 & 3 & & $\mathrm{x}$ & EN & \\
\hline
\end{tabular}

Variables Climáticas. Se utilizaron 19 variables climáticas, derivadas de datos mensuales de precipitación total y temperatura máxima y mínima, tomadas de la base de datos WorldClim (Hijmans et al. 2004; disponibles en http://www.worldclim.org). Las capas de las variables bioclimáticas fueron recortadas para incluir solamente la extensión geográfica de Honduras a una resolución de 0.08333 grados.
Modelo de distribución de especies. Se utilizó MaxEnt (Phillips et al. 2006) por haber mostrado mejores resultados al compararlo con otros métodos (Hernández et al. 2006). Este programa modela la distribución potencial del hábitat idóneo disponible en el área de estudio, en función de su temperatura y precipitación, y de los registros de distribución geográfica de la especie (latitud/longitud) (Phillips et al. 2004, 2006, Phillips 2008). Por lo tanto, la probabilidad 
calculada en MaxEnt es de similitud de hábitat y no de presencia, por lo que el resultado es un mapa de salida en el que cada celda posee un valor de idoneidad de hábitat que, oscila entre 0 y 1.

La evaluación de los modelos obtenidos con MaxEnt fueron evaluados estadísticamente analizando el área bajo la curva (AUC: Area Under the Curve) como una medida del ajuste de los modelos para cada especie (Longoria 2008). Para obtener una distribución de las especies más cercana a la actual y estimar el hábitat potencial conservado y perdido, se editaron los mapas binarios individuales en ArcView 3.2 y ArcGIS 9.3, superponiéndolos con la cobertura del mapa ecológico de Honduras (Holdridge 1962) y el de remanentes (hábitat transformado) de la ecorregión de BS de Honduras (House y Midence 2007b). Los mapas generados se consideraron como los mapas de distribución real para cada especie.

Las áreas de mayor riqueza se obtuvieron al sumar los 27 mapas de distribución real con el fin de obtener un único mapa de riqueza dónde el número máximo corresponde al área con mayor probabilidad de presencia. Por último, se obtuvo un análisis de complementariedad al superponer los 242 registros de presencia de las 27 especies, el mapa de áreas de riqueza total, el mapa de áreas protegidas de Honduras (COHDEFOR-SERNA 1999) y el mapa de remanentes de BS de Honduras de House (2007).

El principio de complementariedad tiene en cuenta a las especies exclusivas para cada sitio y el número total de especies (Zambrano et al. 2009). Relacionando el número de especies en un área $A$, con el número de especies en un área $\mathrm{B}$ y el número de especies en común en $\mathrm{A}$ y $\mathrm{B}$ (Colwell y Coddington 1994). De esta forma, la riqueza total para ambos hábitats combinados es:

$$
S A B=a+b-c
$$

Donde: a es el número de especies del sitio $\mathrm{A}, \mathrm{b}$ es el número de especies del sitio $\mathrm{B}, \mathrm{y} \mathrm{c}$ es el número de especies en común entre los sitios $\mathrm{A}$ y $\mathrm{B}$.

El número de especies únicas a cualquiera de los dos sitios es:

$$
\mathrm{UAB}=\mathrm{a}+\mathrm{b}-2 \mathrm{c}
$$

Finalmente, a partir de estos valores calculamos la complementariedad de los sitios A y B como:

$$
C A B=U A B / S A B
$$

Los valores obtenidos a partir del análisis de complementariedad, varían desde 0 , cuando ambos sitios son idénticos en composición de especies, hasta 1, cuando las especies de ambos sitios son completamente distintas (Colwell y Coddington 1994).

\section{Resultados}

Los mapas de distribución potencial para las 27 especies analizadas en este estudio tuvieron valores de AUC mayores a 0.9, con excepción del mapa generado para la especie Lonchocarpus minimiflorus Donn. Sm, el cual obtuvo una $\mathrm{AUC}=0.827$. Esto sugiere que todos los modelos generados por MaxEnt son consistentes con la realidad y potencialmente útiles para nuestros objetivos.

Las 27 especies en estudio (Cuadro 2), conservan entre el 6 y $30 \%$ de su hábitat. Entre las especies que más hábitat natural han perdido están dos de las siete especies consideradas como vulnerable por la UICN (Leucaena lempirana C.E. Hughes y Lithachne humilis Soderstr), con 6 y $7 \%$ respectivamente de hábitat natural conservado, ambas comparten un hábitat potencial similar pero Leucaena lempirana aún no se ha reportado fuera del Valle del Aguan en el departamento de Yoro y por el contrario Lithachne humilis ha sido reportada para departamentos como Cortés y Olancho pero no para el departamento de Yoro.

Otras especies como Bakeridesia molinae D.M. Bates. con potencial ornamental, muy raro y reportado en estado silvestre entre los 200 y 800 m en bms-T y bs-ST en Francisco Morazán y Yoro, designada por la IUCN como críticamente amenazada, conserva un 11 $\%$ de su hábitat. Tradescantia velutina Kunth. \& C.D. Bouché, considerada como bioindicadora para monitorear los niveles de radiación solar (Cebulska 1992) en el ambiente, conserva solo un $20 \%$ de su hábitat natural (Cuadro 2). Otras especies como Lonchocarpus minimiflorus Donn. Sm, Lonchocarpus phlebophyllus Standl. \& Steyerm, Lonchocarpus retiferus Standl. \& L.O. Williams y Machaerium nicaraguense Rudd, utilizadas en el medio rural para leña, madera y forrajeo, han perdido entre 83 y $90 \%$ de su hábitat natural.

Las 12 especies endémicas estudiadas han perdido entre un 70 a $94 \%$ de su hábitat natural. Entre ellas Coccoloba cholutecensis R.A. Howard es una de las especies más raras de Honduras, críticamente 
Midence Raudales: Áreas Complementarias para la Conservación de la Flora de Preocupación Especial

amenazada que ha sido reportada al noroeste de Tegucigalpa, sobre Cofradía donde además hay registros para otras especies como Pterocaulon pompilianum Standl. \& L.O. Williams, Ipomoea riparum Standl. \& L.O. Williams y Casearia williamsiana Sleumer.

Cuadro 2. Porcentaje de hábitat potencial conservado y perdido restando el área obtenida entre los mapas de distribución potencial con MaxEnt y el mapa de vegetación remanente de bosque seco de House (2007).

\begin{tabular}{|c|c|c|c|c|c|}
\hline Especies & $\begin{array}{c}\text { Hábitat } \\
\text { Original } \\
\left(\mathrm{km}^{2}\right)\end{array}$ & $\begin{array}{c}\text { Hábitat } \\
\text { Conservado } \\
\left(\mathrm{km}^{2}\right)\end{array}$ & $\begin{array}{c}\text { Hábitat } \\
\text { Perdido } \\
\left(\mathrm{km}^{2}\right)\end{array}$ & $\begin{array}{l}\text { Hábitat } \\
\text { Conser- } \\
\text { vado (\%) }\end{array}$ & $\begin{array}{c}\text { Hábitat } \\
\text { Perdido } \\
\text { (\%) }\end{array}$ \\
\hline Agonandra macrocarpa L.O. Williams & 12,780 & 1,497 & 11,283 & 11.71 & 88.29 \\
\hline Anisacanthus tetracaulis Leonard & 3,632 & 665 & 2,967 & 18.31 & 81.69 \\
\hline Bakeridesia molinae D.M.Bates & 14,428 & 1,516 & 12,912 & 10.51 & 89.49 \\
\hline Calliandra molinae Standl. & 3,163 & 817 & 2,346 & 25.83 & 74.17 \\
\hline Casearia williamsiana Sleumer & 9,260 & 1,297 & 7,963 & 14.00 & 86.00 \\
\hline Coccoloba cholutecensis R.A. Howard & 11,545 & 1,433 & 10,112 & 12.41 & 87.59 \\
\hline Croton comes Standl. \& L.O. Williams & 4,662 & 1,048 & 3,614 & 22.48 & 77.52 \\
\hline Guettarda sageretioides Ant. Molina & 4,530 & 878 & 3,652 & 19.38 & 80.62 \\
\hline Hechtia malvernii A.J. Gilmartin & 11,038 & 1,450 & 9,588 & 13.14 & 86.53 \\
\hline Ipomoea riparum Standl. \& L.O. Williams & 4,331 & 921 & 3,410 & 21.27 & 78.73 \\
\hline Justicia calliantha Leonard & 2,655 & 788 & 1,867 & 30.00 & 70.00 \\
\hline Leucaena lempirana C.E. Hughes & 10,471 & 614 & 9,857 & 5.86 & 94.14 \\
\hline Lithachne humilis Soderstr. & 11,228 & 757 & 10,471 & 6.74 & 93.25 \\
\hline Lonchocarpus minimiflorus Donn. Sm & 9,034 & 1,370 & 7,664 & 14.61 & 84.84 \\
\hline Lonchocarpus phlebophyllus Standl. \& Steyerm & 9,514 & 1,320 & 8,194 & 13.87 & 86.13 \\
\hline Lonchocarpus retiferus Standl. \& L.O. Williams & 11,288 & 1,115 & 10,173 & 9.88 & 90.12 \\
\hline Lonchocarpus sanctuarii Standl. \& L.O. Williams & 2,888 & 527 & 2,371 & 18.25 & 81.75 \\
\hline Lonchocarpus trifolius Standl. \& L.O. Williams & 4,975 & 924 & 4,051 & 18,57 & 81.43 \\
\hline Lonchocarpus vallicola (Stand. \& F.J.Herm.) M. Sousa & 1,095 & 312 & 783 & 28.49 & 71.51 \\
\hline Machaerium nicaraguense Rudd & 5,680 & 988 & 4,692 & 17.39 & 82.61 \\
\hline Myrmecophila wendlandii (Rchb.f.) G.C Kenn & 6,759 & 1,152 & 5,607 & 17.04 & 82.96 \\
\hline Nopalea lutea Rose & 12,468 & 1,564 & 10,904 & 12.54 & 87.46 \\
\hline Opuntia guatemalensis Britton \& Rose & 9,150 & 1,243 & 7,907 & 13.58 & 86.42 \\
\hline Pereskia lychnidiflora DC. & 6,214 & 1,154 & 5,060 & 18.57 & 81.43 \\
\hline Pterocaulon pompilianum Standl. \& L.O. Williams & 3,630 & 896 & 2,734 & 24.68 & 75.32 \\
\hline Stenocereus yunckerii Rose & 8,707 & 725 & 7,982 & 8.33 & 91.67 \\
\hline Tradescantia velutina Kunth. \& C.D. Bouché & 5,033 & 1,002 & 4,031 & 19.91 & 80.09 \\
\hline
\end{tabular}

Con las especies con categoría CITES II, cactáceas como Nopalea lutea Rose, Opuntia guatemalensis Britton \& Rose, Pereskia lychnidiflora DC y Stenocereus yunckerii (Standl.) M. Bravo \&
Sánchez-Mej. han perdido entre 81 y $92 \%$ de su hábitat natural. De este grupo, el más afectado por tener un intervalo de distribución limitado a la región noreste de Honduras es Stenocereus yunckerii a pesar 
de ser una especie bandera y hábitat para las especies emblemáticas el colibrí esmeralda (Amazilia luciae) y el reptil endémico (Ctenosaura melanosterna), junto a otras especies endémicas como Pilosocereus chysacanthus, Nopalea hondurensis, Eugenia coyolensis, Lonchocarpus yoroensis, Leucaena lempirana, Dioon mejiae y Zamia standleyi solamente conserva $8 \%$ de su hábitat natural. Myrmecophila wendlandii (Rchb.f.) G.C Kenn es considerada una especie de orquídea indicadora de zonas semiáridas, ya solo conserva $17 \%$ de su hábitat natural en valles como Aguan, Agalta, Morcelí, Texiguat y Oropolí.

Áreas de mayor riqueza para la ecorregión de BS en Honduras. Al sumar los 27 mapas de distribución real para obtener un único mapa de riqueza (Figura 1). se observó que las áreas de mayor riqueza con hábitat potencial para 19-27 especies fueron todos los BS de los valles del interior entre 600 y $800 \mathrm{msnm}$, en la región central de Honduras calificados por Holdridge dentro del bs-ST y que son fragmentos en el valle de Comayagua en los departamentos de La Paz y Comayagua y en Francisco Morazán sobre el valle de Tegucigalpa y Talanga (Cuadro 3), este es el único ecosistema de BS que no está representado en el Sistema Nacional de Áreas Protegidas de Honduras (SINAPH). Otros sitios importantes son los bs-T en los departamentos de El Paraíso, Olancho y Yoro, sobre los 350 y 700 msnm.

El Paraíso es el departamento que aún conserva más áreas de riqueza dentro del bs-T, tiene 19 de las 27 especies, sobre todo en los valles de Morocelí y Oropolí que descienden hasta los municipios sureños de Duyure y Morolica en Choluteca. Otra área importante está en Olancho donde apenas se registran algunos fragmentos de hábitat natural muy pequeños en un área de 247 ha con varias especies para conservar ( $\geq 19$ especies) sobre el valle de Lepaguare que comprende el municipio de San Francisco de la $\mathrm{Paz}$, adentro del monumento natural conocido como Boquerón y que forman parte del SINAPH. Finalmente, solamente existe un área conservada para el bms-T al norte del departamento de Yoro adentro de la reserva del colibrí esmeralda (1300 ha) sobre el valle de Aguan.

Complementariedad. Se visualizaron cuatro regiones con 15 especies: Comayagua, Tegucigalpa, Morocelí y Oropolí (Cuadro 3). Basado en el principio de complementariedad se seleccionaron tres áreas: Oropolí (4 especies), Tegucigalpa (cuatro especies) y Morocelí (tres especies) (Figura 2), el resto de las especies reportadas aparecen actualmente dispersas en áreas transformadas a sistemas productivos. Según el índice de complementariedad cada localidad tiene una distribución de especies únicas (Cuadro 4).

\section{Discusión}

En Honduras no solo las especies están en peligro, sino también ecosistemas completos (House y Midence 2007a). En general, la ecorregión de BS en Honduras ha perdido $17,708.14 \mathrm{~km}^{2}$ entre 1962 y 2007, una pérdida de hábitat de aproximadamente el $90 \%$ para las 27 especies en estudio. Las especies consideradas antes como de amplia distribución sobre el bs-T, están restringidas ahora principalmente en el sur del país sobre la planicie costera del Pacifico y en algunos de los valles más grandes como los de Olancho y Cortés. En estas áreas apenas sobreviven algunos fragmentos de un Bosque semideciduo alto con árboles de hasta $40 \mathrm{~m}$. en fragmentos aislados muy intervenidos y a lo largo de quebradas sobre terraplenes escarpados (Cerrato et al. 2002).

En nuestro estudio se pudo observar que el BS donde existió un mayor traslape ( $\geq 19$ especies) de especies fue sobre los valles interiores de la zona central y parte del valle de Oropolí. Este hecho tiene serias implicaciones en conservación debido a la rápida expansión urbana hacia la periferia y la conversión del suelo a otros sistemas productivos en esas zonas. Estas actividades ponen en riesgo hábitats con una alta concentración de endemismo, agravado por el hecho de que apenas unos fragmentos $(\leq 5 \mathrm{~km} 2)$ de los $17,708.14 \mathrm{Km} 2$ de BS mapeado por House (2007) se encuentra adentro del SINAPH sobre el Valle de Lepaguare.

Las tres mejores áreas según el principio de complementariedad resultaron ser: Oropolí $(27,955$ ha), Tegucigalpa $(10,200$ ha) y Morocelí (3,813 ha). Estas áreas en la zona centro y sur de Honduras podrían funcionar como conexión entre reservas privadas, estatales y corredores biológicos.

Se espera que con la intervención y un manejo técnico y administrativo adecuado, estos hábitats funcionen como áreas semilleros para restaurar áreas degradadas, donde su conservación requiera necesariamente la restauración a partir de los fragmentos remanentes de mayor riqueza para aumentar la riqueza de especies y la complejidad de la comunidad a nivel estructural y funcional. 


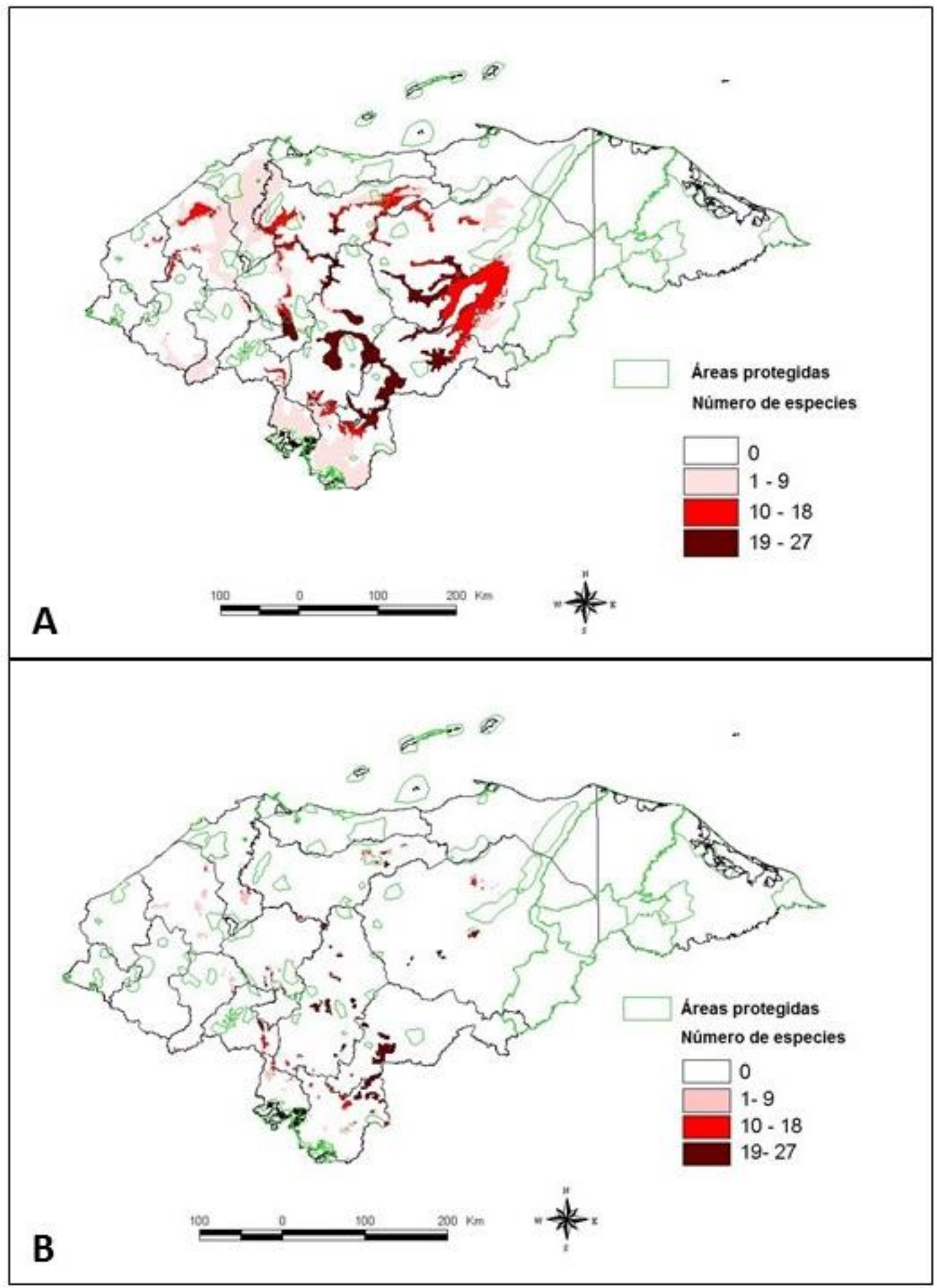

Figura 1. Áreas con mayor riqueza de especies considerando 27 mapas de distribución editados con A el mapa ecológico de Honduras (Holdridge 1962) y B el mapa de remanente de bosque seco (House 2007). 
Cuadro 3. Especies presentes en las áreas de mayor riqueza de 27 especies del bosque seco para Honduras seleccionadas para este estudio

\begin{tabular}{llc}
\hline Región & Especies & $\begin{array}{c}\text { Número de } \\
\text { especies }\end{array}$ \\
\hline Aguan & Bakeridesia molinae D.M.Bates & 1 \\
\hline Comayagua & $\begin{array}{l}\text { Hechtiamalvernii A.J.Gilmartin } \\
\text { Lonchocarpus minimiflorus Donn. Sm } \\
\text { Agonandra macrocarpa L.O. Williams }\end{array}$ & 3 \\
\hline \multirow{3}{*}{ Tegucigalpa } & $\begin{array}{l}\text { Bakeridesia molinae D.M. Bates } \\
\text { Coccoloba cholutecensis R.A. Howard } \\
\text { Guettarda sageretioides Ant. Molina } \\
\text { Casearia williamsiana Sleumer }\end{array}$ & 4 \\
\hline \multirow{3}{*}{ Morocelí } & $\begin{array}{l}\text { Lonchocarpus vallicola (Stand. \&F.J. Herm.)M. Sousa } \\
\text { Tradescantia velutina Kunth. \& C.D. Bouché }\end{array}$ & 3 \\
\hline Oropolí & Machaerium nicaragüense Rudd & \\
& Lonchocarpus trifolius Standl. \& L.O. Williams & 4 \\
& Opuntia guatemalensis Britton \& Rose \\
& Lonchocarpus phlebophyllus Standl. \& Steyerm \\
& Myrmecophila wendlandii (Rchb.f.) G.C Kenn & 15 \\
\hline
\end{tabular}

Cuadro 4. Análisis de Complementariedad para tres regiones de bosque seco en Honduras, $a=$ Oropolí, $b=$ Tegucigalpa, c=Morocelí.

\begin{tabular}{lccc}
\hline Índice de complementariedad & $(\mathbf{a}, \mathbf{b})$ & $\mathbf{( a , c )}$ & $\mathbf{( c , b )}$ \\
\hline Número de especies de la primera localidad de cada comparación $(\mathrm{A})$ & 4 & 4 & 3 \\
\hline Número de especies de la primera localidad de cada comparación $(\mathrm{B})$ & 4 & 4 & 3 \\
\hline Especies en común entre los dos sitios ( C ) & 0 & 0 & 0 \\
\hline Especies exclusivas sitio A & 4 & 4 & 3 \\
\hline Especies exclusivas sitio B & 4 & 3 & 4 \\
\hline $\mathrm{SAB}=\mathrm{a}+\mathrm{b}-\mathrm{c}$ & 8 & 7 & 7 \\
\hline $\mathrm{UAB}=\mathrm{a}+\mathrm{b}-2 \mathrm{c}$ & $\mathbf{7}$ & $\mathbf{7}$ \\
\hline Complementariedad AB=UAB $\div \mathrm{SAB}$ & $\mathbf{1}$ & $\mathbf{1}$ & $\mathbf{1}$ \\
\hline
\end{tabular}

Finalmente, es indispensable verificar en el campo los sitios potenciales de distribución de las especies en estudio, evaluar rápidamente la biodiversidad para aumentar la información que se tiene hasta ahora en las colecciones, sobre todo de especies de interés, y con esto mejorar la calidad de los MDE, impulsar estudios fenológicos, genéticos y poblacionales en las áreas recomendadas. Por último, se recomienda reevaluar el estado de conservación para las 27 especies en estudio.

Agradecimiento. De manera particular a la Dra. Teresa Patricia Feria la cual contribuyó significativamente con su asesoramiento y a la Lic. Thelma Mejía Ordóñez por la revisión del document. 


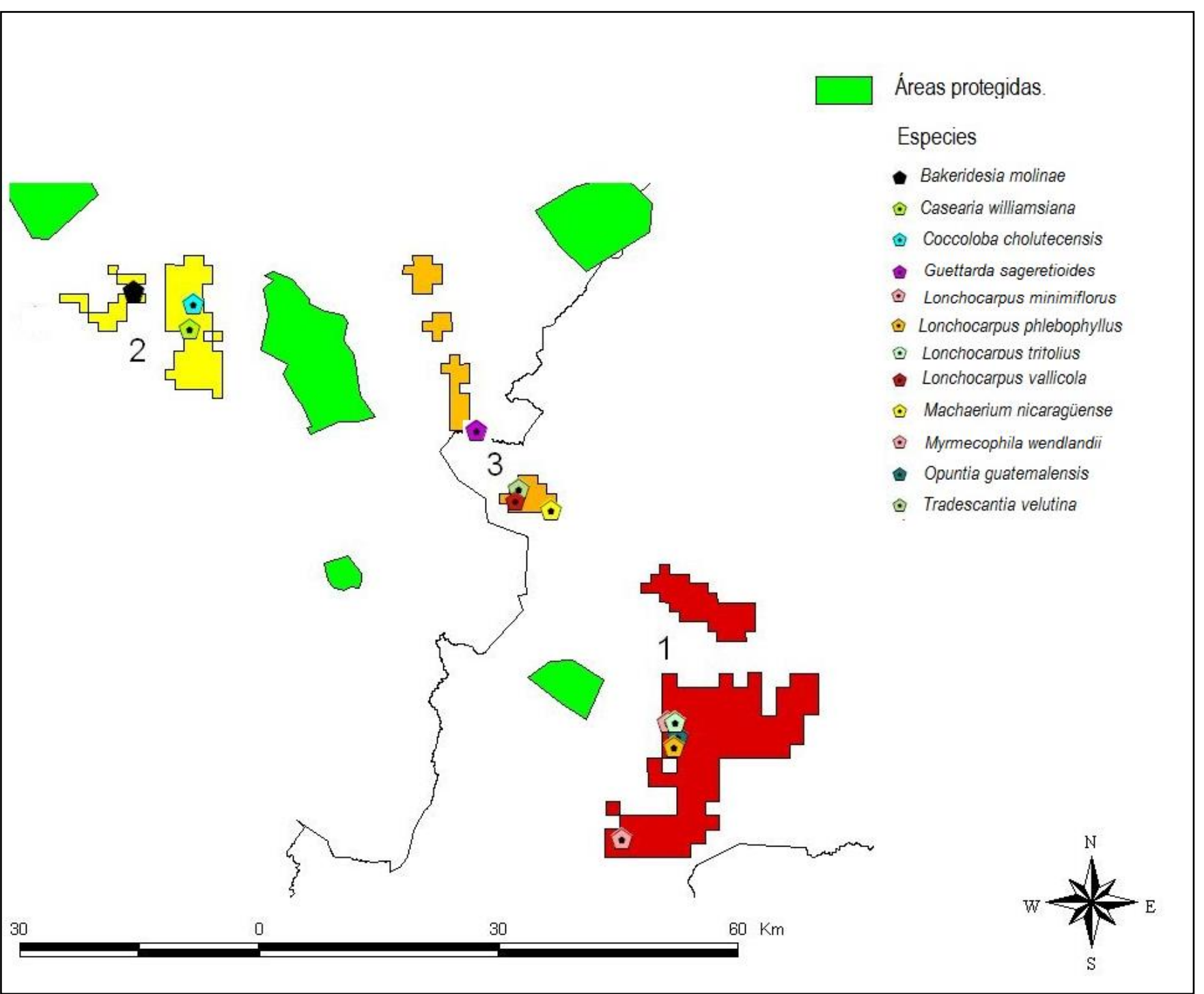

Figura 2. Áreas complementarias para la conservación del bosque seco en Honduras basadas en el mapa de remanentes de bosque seco (House y Midence 2007b) según orden de prioridad: Oropolí=1, Tegucigalpa=2, Morocelí=3.

\section{Literatura Citada}

Agudelo, N. 1987. Ecosistemas terrestres de Honduras. Asociación Hondureña de Ecología. Honduras. $16 \mathrm{p}$.

Cebulska, A.1992. Tradescantia stamen-hair mutation bioassay on the mutagenicity of radioisotopecontaminated air. Mutation Research 270 (1): 23-29.

Cerrato, C., P. House y D. Vreugdenhil. 2002. Racionalización del Sistema de Áreas Protegidas de Honduras. Volumen IV: Especies de preocupación especial. PROBAP/WB/UNDP/GEF. Honduras. 16 p.
Colwell, R. y J. Coddington. 1994. Estimating terrestrial biodiversity through extrapolation. Philosophical Transactions of the Royal Society of London 345 (1311): 101-118.

Garcia, J., J.I. Restrepo, S. Zavala y S. Triminio. 2006. Preparación de una estrategia para la conservación de especies crítica y globalmente amenazadas en Mesoamérica Norte. Memoria del taller. Centro Zamorano de Biodiversidad. Zamorano, Honduras. p 27. 
Hernández, P., C. Graham, L. Master y D. Albert. 2006. The effect of sample size and species characteristics on performance of different species distribution modeling methods. Ecography 29: 773-785.

Hijmans, R., L. Guarino, C. Bussink, P. Mathur, M. Cruz, I. Barrantes y E. Rojas. 2004. DIVA-GIS,versión 4. A geographic information system for theanalysis of biodiversity data. Manual. (Disponible enhttp://www.diva-gis.org)

Holdridge, L. 1962. Mapa Ecológico de Honduras, 1:1, 000,000. Tropical Science Center, San José, Costa Rica. 1 mapa.

House, P.R. y C.M. Midence. 2007a. Distribución de especies prioritarias. Informe técnico DiBio-TNC. Honduras. p. 20.

House, P.R. y C.M. Midence. 2007b. Identificación de Áreas Prioritarias para la conservación de ecosistemas secos y semiáridos de Honduras. Informe Técnico TNC. Honduras. p. 53.

Longoria, C.A. 2008. Distribución hipotética de especies de aves y mamíferos con categoría de riesgo en la NOM059-ECOL-2001 registradas en el estado de Durango. Tesis. México. p. 79.

Maass, J.M., H.D. Mooney y E. Medina. 1995. Conversion of tropical dry forest to pasture and agriculture. Cambridge University Press. Nueva York. 399-422.

Marshall, LG., S.D. Webb, S.D. Sepkoski y D.M. Raup (1982) Mammallan Evolution and the Great American Interchange. Science 251:1351-57.

Mejía, T.M. y P.R. House. 2008. Especies de Preocupación Especial en Honduras. Informe de Consultoría para SERNA. Tegucigalpa. $59 \mathrm{p}$.

McGough, H., M. Groves, M. Mustard y C. Brodie. 2004. CITES y las plantas: Guía del usuario. Royal Botanic Gardens, Kew, Reino Unido. 67 p.
Midence C.M. 2010. Identificación de áreas prioritarias para la conservación de Ecosistemas semiaridos de Honduras. Tesis de Maestria. Universidad Internacional de Andalucía. Huelva, España. $104 \mathrm{p}$

Miles, L., A. C. Newton, R.S. DeFries, C.M. Ravilious, I. Blyth, S. Kapos y J.E. Gordon. 2006. A global overview of theconservation status of tropical dry forests. Journal of Biogeography 33:491-505.

Murphy, P.G. y A.E. Lugo. 1995. Dry Forests of Central America and the Caribbean. Cambridge University Press, Cambridge. p. 9-34.

Phillips, S.J., R.P. Anderson y R.P. Schapire. 2006. Maximum entropy modeling of species geographic distributions. Ecological Modelling 190 (3/4): 231-259.

Phillips, S.J., M. Dudík y R.E. Schapire. 2004. A maximum entropy approach to species distribution modeling. ACM Press. Canadá. p. 655-662.

Phillips, S.J. 2008. Transferability, sample selection bias and background data in presence-only modeling: A response to Peterson et al. (2007). Ecography 31: 272278.

Pineda, N. 1997. Geografía de Honduras. Editorial Guaymuras. Honduras. 494 p.

Portillo, H.O. 2007. Recopilación de la Información Sobre la Biodiversidad de Honduras. Informe Final de la Consultaría. INBIO-DiBio. Honduras. 118 p.

Prado, D.E. y P.E.Gibbs. 1993. Patterns of species distribution in the dry seasonal forests of South America. Annals of the Missouri Botanical Garden 80:902-927.

Zambrano, G. y G. Ortiz. 2009. Diversidad de Lepidópteros diurnos en tres localidades del corredor biológico y multicultural Munchique-Pinche, Cauca, Colombia. Boletín científico museo historia natural 13 (1): 214.

Recibido para publicación el 15 de marzo del 2016.

Aceptado para publicación el 10 de diciembre de 2016. 